\title{
Germanica
}

\section{La technique narrative dans le roman de Theodor Plievier Der Kaiser ging, die Generäle blieben}

Die Erzähltechnik in Theodor Plieviers roman Der Kaiser ging, die Generäle blieben

Pierre Vaydat

\section{OpenEdition}

\section{Journals}

Édition électronique

URL : http://journals.openedition.org/germanica/2207

DOI : 10.4000/germanica.2207

ISSN : 2107-0784

\section{Éditeur}

Université de Lille

\section{Édition imprimée}

Date de publication : 1 décembre 1994

Pagination : 13-29

ISSN : 0984-2632

\section{Référence électronique}

Pierre Vaydat, «La technique narrative dans le roman de Theodor Plievier Der Kaiser ging, die Generäle blieben », Germanica [En ligne], 14 | 1994, mis en ligne le 20 janvier 2014, consulté le 06 octobre 2020. URL : http://journals.openedition.org/germanica/2207 ; DOI : https://doi.org/10.4000/germanica.2207

Ce document a été généré automatiquement le 6 octobre 2020.

(c) Tous droits réservés 


\title{
La technique narrative dans le roman de Theodor Plievier Der Kaiser ging, die Generäle blieben
}

\author{
Die Erzähltechnik in Theodor Plieviers roman Der Kaiser ging, die Generäle \\ blieben
}

Pierre Vaydat

1 Du point de vue de l'esthétique narrative, le roman Der Kaiser ging, die Generäle blieben constitue un cas limite à deux égards. Il s'agit d'abord d'une reconstitution événementielle utilisant la technique du reportage, dans laquelle la part de la fiction (atmosphère, suspense) est très réduite. Ensuite, le lecteur se demande s'il est en face d'un roman d'actualité, d'un «Gegenwartsroman» au sens strict, ou s'il faut considérer l'ouvrage comme un roman historique, étant donné qu'il a été écrit durant la phase terminale de la République de Weimar (il est paru au Malik-Verlag en 1932) et qu'il relate par contre les événements révolutionnaires de novembre 18, ceux qui ont donné naissance à ladite République. La question à laquelle on va tenter de répondre est la suivante: quel est, dans cette œuvre, le rapport de la création esthétique au vécu politique? En quoi le traitement fictionnel, ou plus précisément l'imitation de la mimésis ${ }^{1}$, confère-t-il à ce vécu un degré supérieur d'intelligibilité ?

2 Le point de départ de l'analyse sera le choix même de l'auteur. Pourquoi a-t-il opté pour une présentation romancée, pour l'artifice littéraire, de préférence à une présentation historique? Pourquoi le recours à la littérarité, en l'occurrence aux techniques narratives du réalisme, qui génèrent l'illusion du réel, alors que le réel (c'est-à-dire l'effet de réel) est constitué ici d'emblée comme vraisemblance maximale par le concordance des très nombreux documents d'époque ? Cette question revêtait aux yeux de Plievier lui-même une importance telle qu'il a éprouvé le besoin de rédiger une postface (Nachwort) où il légitime le caractère hybride de son récit, qui est à la fois récit de fiction et récit historique :

Ich gab diesem Versuch, Geschichte zu schreiben, Romanform, weil ich glaubte,

dass Ereignisse, die nicht durch den Austausch von diplomatischen Noten, sondern 
durch den Zusammenprall gegensätzlicher Kräfte ausgelöst wurden, sich der rein wissenschaftlichen Darstellung entziehen. Man mag noch so viele Daten aus einer Zeit zusammentragen, - ein lebendiges Gesamtbild wird erst durch die künstlerische Gestaltung ergeben².

Etudier les procédés de cette "figuration", de cette mise en récit, est donc une investigation à laquelle nous incite l'auteur en personne. La mise en récit doit conduire, au-delà d'une exigence scrupuleusement respectée d'authenticité factuelle, à une vérité d'ordre supérieur débordant la simple exactitude historique. Telle est ici la visée première de ce que Paul Ricœur appelle dans Temps et Récit la « refiguration ».

La postface nous permet d'entrevoir aussi la réponse à l'autre question; roman historique ou roman d'actualité ? ${ }^{3}$ Elle apporte les éclaircissements suivants :

Die politische Haltung grosser Teile des deutschen Volkes, insbesondere der Jugend, zur Frage des verlorenen Krieges und der Entstehung der Republik beweist: die geschichtlich so bedeutenden Ereignisse im Herbst 1918 sind weiten Kreisen unbekannt geblieben, zumindest ist die Erinnerung an jene Zeit durch falsche Darstellungen verschüttet worden. Im vorliegenden Band habe ich versucht, eine umfassende Schilderung jener Wochen zu geben, in denen die Grundlagen für Deutschlands Existenz nach dem Kriege gelegt wurden.

5 Point de doute: pour Plievier, la reconstitution de ces semaines décisives et fondatrices, y compris et même surtout au sens négatif, est un sujet de pure actualité. De la réussite du projet artistique exposé ci-dessus, de la mise en scène et en récit de l'histoire allemande la plus récente, dépend l'impact de la démonstration antifasciste sur une opinion de plus en plus mécontente du régime républicain (ou de ce qui en reste après 1930) et qui est en train de réagir en tournant le dos à la démocratie. Il s'agit de donner vie au passé immédiat pour illuminer le présent, mais aussi pour le transmuter en champ d'action où pourront se déployer des forces démocratiques rendues enfin conscientes des raisons véritables de leur carence. Or, à cette époque encore, le roman est le moyen privilégié de la communication de masse, alors qu'une œuvre à visée purement historique ou même un éditorial fulgurant ne touchent qu'un nombre restreint de lecteurs, et surtout ne déclenchent aucune dynamique émotionnelle. Il s'agit de mobiliser, à l'aide d'un roman digne d'atteindre les tirages phénoménaux de Im Westen nichts Neues, ce que M. Winock appelle le «sentiment de l'urgence civique ", et cela à un moment où la désaffection à l'égard des valeurs républicaines paralyse les velléités de résistance à la percée nazie.

6 Der Kaiser blieb, die Generäle blieben fait partie d'un cycle romanesque resté inachevé. Cinq romans étaient en projet. Deux seulement ont paru: Des Kaisers Kulis (1930) et le texte qui fait l'objet de la présente étude. Du troisième roman, qui devait mener le lecteur jusqu'en 1920, Plievier n'a pu sauver en partant pour l'exil que trois chapitres ${ }^{4}$.

7 La période recréée par l'écriture s'étend du 16 octobre 1918 au soir du 9 novembre. Elle trouve son unité d'atmosphère et d'action, et le roman trouve du même coup sa donnée dramatique, dans la situation chaque jour aggravée de l'Allemagne durant ces dernières semaines de la première guerre mondiale. Les événements se succèdent si vite, leur pression sur les actants augmente à un rythme tel, que les processus de décision fonctionnent de moins en moins normalement et font place, bientôt, à une série d'improvisations. Il n'y a plus de maîtrise de l'événement, donc plus de stratégie politique pensable. L'agir prend la forme d'une série de choix tactiques dictés par des circonstances en continuel bouleversement. La perception des suites possibles est confuse. Le rendu de cette relation au temps, de cette perception brouillée par 
l'accélération soudaine du flux événementiel, voilà un autre privilège de l'art narratif qui confère au récit du scripteur littéraire sa supériorité par rapport au récit historique. L'instauration d'une temporalité narrative est incompara-blement plus parlante qu'une chronologie commentée. Elle permet de faire comprendre au "narrataire », c'est-à-dire au lecteur contemporain présumé démocrate, que c'est la médiocrité des acteurs, leur passivité fondamentale, l'absence d'un projet révolutionnaire cohérent, qui leur font concevoir toutes les conséquences possibles du changement en cours comme des suites forcément dévastatrices. C'est le sentiment qu'une chance unique de démocratisation est en train d'être irrévocablement gâchée qui légitime l'emploi de la technique simultanéiste. Parfaitement mise en œuvre, elle ne produit pas un effet d'éparpillement, mais fait apparaître au contraire l'unité et la continuité du processus. La logique diégétique est celle de la fatalité, puisque les actants renoncent, sous divers prétextes, à exercer leur liberté, ou en font, comme l'USPD et les spartakistes, un usage erroné.

8 Le lecteur contemporain avait présents à l'esprit les faits saillants que le texte constitue en configuration actantielle: dissolution de la monarchie militaire prusso-allemande (ou plutôt de ce qui en subsiste, car la dictature exercée en fait par Ludendorff s'y est substituée depuis 1917); débâcle menaçante sur le front; échec de la mise en place d'une monarchie constitutionnelle. Le gouvernement du prince Max de Bade, auquel participent les socialistes majoritaires, est privé soudainement de toute autorité lorsqu'éclate la révolte des marins de Kiel, relayée et amplifiée par le mouvement des «Revolutionäre Obleute» et les militants spartakistes. La course au pouvoir s'engage entre les socialistes indépendants et les socialistes majoritaires; ces derniers sont en fait, et contrairement à l'idéologie qu'ils prônent, le parti de l'ordre. Dès lors, Ebert est acculé à l'alliance avec le général Groener, conclue par téléphone au soir du 9 novembre. Le moment où Ebert, ayant décroché l'appareil, entend la voix de Groener, constitue la clôture du livre. L'un des effets recherchés est l'effet de reconnaissance. La mise en récit opère la donation de sens dont l'histoire vécue a frustré le lecteur ; elle exerce par conséquent une fonction libératrice, bien qu'elle débouche sur le péché originel de la République.

9 La pression croissante des événements assure d'elle-même la progression dramatique du récit. Tout est raconté au présent. Les notations de temps, les indications de lieu sont laconiques ${ }^{5}$. Le rythme narratif est précipité. L'auteur (le récitant) commente le sens de chaque événement, une fois celui-ci raconté. Presque à chaque page est affirmé le but didactique. Dans la conscience du lecteur, qui vit chaque jour, en ce début des années 30, la dissolution de Weimar, chaque épisode se met en place dans un schéma interprétatif, formant un chaînon dans l'explication génétique de la catastrophe. L'intention démonstrative est patente, la voix du narrateur omniscient n'en fait aucun mystère. La leçon de chaque unité narrative est la même: nous constatons la « redondance » qui, selon S. Suleiman ${ }^{6}$, caractérise le roman à thèse.

Les personnages peuvent être répartis en trois catégories.

11 D'abord, les personnages historiques, les acteurs réels : Groener, Ludendorff, Max von Baden, Ebert, Scheidemann, Liebknecht, Noske, etc.

12 Puis des personnages fictifs, figurant des destins individuels typiques, mais à qui fait évidemment défaut la richesse psychologique indispensable pour être de véritables personnages romanesques, ceux que l'on rencontre dans le roman de formation comme dans le roman-parabole. La technique du roman-reportage exclut l'intériorisation du 
monde représenté, de même qu'elle en prohibe pratiquement la représentation symbolique. On peut distinguer parmi ces personnages inventés ceux qui agissent, comme les marins de Kiel, ou, dans le sens contraire, le contremaître Karl Lange, obstinément fidèle au SPD ; et ceux qui représentent emblématiquement les victimes : le soldat Max Müller, écrasé dans son trou par un tank (c'est la première scène du livre); sa femme Trude Müller, qui vit pauvrement dans une Mietskaserne et dont l'enfant, souffrant de malnutrition et d'une pneumonie, va également mourir. Ces personnages vivent l'Histoire comme un drame personnel. Ils sont ceux auxquels le lecteur populaire peut s'identifier et par lesquels s'abolit donc la distance instaurée obligatoirement par la méthode documentaire, distance génératrice de détachement scientifique et, par voie de conséquence, de déshumanisation. Les personnages fictifs permettent de faire appel à la sensibilité du lecteur; celui-ci découvre en eux les véritables héros, par opposition aux grandes figures dont l'historiographie a retenu les noms. La pseudo-objectivité de la narration purement historique ou se voulant telle ne peut rendre en effet ce qui demeure le privilège de l'art littéraire : faire éprouver en utilisant les conventions du genre romanesque l'énorme intervalle qui sépare ceux que l'historien considère comme des grands hommes du peuple souffrant voué à l'anonymat.

Troisièmement, les masses, représentées tantôt par des personnages individualisés (comme ceux qui viennent d'être évoqués), tantôt saisies dans leur flux et dans leurs élans par des procédés de description dont quelques uns peuvent être qualifiés de cinématographiques.

Il n'y a pas de personnage central. Il n'y a pas non plus de personnages vraiment individuels. Tous, l'instance d'énonciation mise à part, ont le même statut sémiologique : ce sont des « personnages-référentiels ».

La technique narrative utilisée par Plievier est celle de la «Neue Sachlichkeit». Elle comporte principalement quatre innovations : a) le reportage personnalisé, autrement dit la figuration du vécu personnel d'un actant dans une situation évoquée au présent narratif par les procédés du reportage, c'est-à-dire par une saisie de l'objet à partir de l'apparence et la mise à nu, ensuite, de son fonctionnement; b) l'implication fictionnelle du lecteur dans les processus de décision exposés minutieusement, les réflexions et les réactions des personnages réels étant rapportés ou reconstitués en style indirect libre, alternant avec la relation dialoguée des réunions ; c) le montage du flux événementiel en séquences cinématographiques (une succession d'unités narratives assez courtes, épisodes dont le lieu est chaque fois indiqué avec une certaine précision topographique et figuré par un décor minimal, celui d'un "plan» ou de plusieurs); aux variations d'intensité dramatique dans la conduite du récit correspondent des focalisations internes diverses; d) la renonciation à la symbolique, c'est-à-dire la suppression de tout rapport entre l'action et une quelconque transcendance ; le monde de l'œuvre est celui de l'existence nue, dans lequel la liberté de l'homme est l'obligation d'agir pour transformer sa condition; le monde, en somme, de la responsabilité intégrale de l'homme, sans alibi et sans intercession mythique possible.

16 Le sujet de Der Kaiser ging, die Generäle blieben est l'échec de l'apprentissage révolutionnaire collectif. Le lecteur est censé tirer la leçon de cet échec pour lui-même, en prenant conscience de son histoire vécue, mais subie, jamais maîtrisée. On peut appliquer au récit de Plievier ce que Susan Suleiman dit de L'Espoir de Malraux : 
En 1937, le «bon » lecteur de L'Espoir était quelqu'un qui se transformait au cours de sa lecture en acteur véritable : il devenait un personnage dans une « histoire vraie » dont la fin n'était pas encore écrite ${ }^{7}$. 
Pendant le trajet, il reçoit des télégrammes dont les extraits cités montrent que les Allemands reculent, tout en se défendant avec acharnement.

Épisode III (17.10.1918) : Réunion du gouvernement à la Chancellerie du Reich, sous la présidence $d u$ chancelier Max von Baden. 1. Ludendorff arrive à la Chancellerie. On le voit descendre de voiture et entrer dans le bâtiment. 2. La réunion. Ludendorff tente d'obtenir des renforts supplémentaires; il réclame 600000 hommes. Mais la compétence qu'il étale n'en impose plus à ses interlocuteurs. Le pouvoir militaire a cessé de subjuguer le pouvoir civil, dès lors qu'il ne peut plus se targuer d'être victorieux. Intervention de l'auteur: bref rappel du programme de la nation soldatique que Ludendorff rêve d'appliquer après la guerre. Cette utopie a déjà coûté au peuple allemand 1800000 morts. Pour l'instant, l'appui des sociaux-démocrates est pour Ludendorff le dernier espoir. Il demande à Scheidemann et à Ebert de galvaniser la population pour un ultime sursaut. Soif rappelle alors opportunément que c'est le Haut-Commandement luimême, la OHL, qui a fait pression sur le pouvoir civil pour qu'une demande d'armistice soit faite au plus vite, et cela début octobre. Ludendorff déclarait impossible, souligne Soif, la résistance prolongée qu'il préconise maintenant. Le prince Max de Bade qualifie les propos de Ludendorff de tentative de manipulation. Portrait du prince Max de Bade: un grand aristocrate humaniste, mais que son "impérialisme éthique ", doctrine nébuleuse, a conduit à se placer sans remords au service de la machine de guerre allemande. Il est chargé à présent de négocier l'armistice et de sauver ce qui peut l'être d'un régime aux abois.

Épisode IV (22.10.1918). Séance houleuse au Reichstag. 1. Le prince Max de Bade paraît au Richstag. 2. Scheidemann, qui se préparait à prendre place dans l'hémicycle, a été retenu dans le hall. Il est informé que Karl Liebknecht, libéré de prison la veille, appelle les populations à exiger la fin immédiate de la guerre, la démission du gouvernement et la révolution sociale. 3. La scène est de nouveau dans l'hémicycle. Le prince Max de Bade rend compte aux députés de l'état d'avancement des pourparlers avec l'Entente. Il exhorte à la résistance au cas où les conditions posées seraient trop dures. Applaudissements des conservateurs. Mais les partis majoritaires (USPD, SPD, libéraux, Centre catholique) clament leur indignation. Intervention de l'auteur: s'ils désapprouvent cette attitude, ce n'est pas par désir sincère de paix, puisqu'ils ont soutenu le gouvernement en toutes circonstances, allant jusqu'à entériner le brutal traité de Brest-Litovsk (il n'est pas fait référence à la résolution de paix de 1917) ; mais c'est par électoralisme. Le peuple leur a signifié qu'il était à bout de forces. Le prince Max de Bade tente d'apaiser la gauche en promettant de procéder d'un seul coup à toutes les réformes démocratiques que la monarchie prusso-allemande avait obstinément refusées jusqu'à présent. Intervention de l'auteur: retour en arrière, rappel des circonstances qui ont amené la scission du mouvement socialiste allemand. Reprise du récit : une fois de plus, les socialistes majoritaires apportent leur soutien au gouvernement impérial. Ebert, le tacticien, prend la parole. Il fait un pas en direction des socialistes indépendants, dénonçant la responsabilité de la dictature militaire de fait exercée par la OHL, qui veut maintenant se décharger sur les civils de la responsabilité de la défaite. Discours du ministre de la guerre. Il est interrompu à plusieurs reprises par des clameurs et des rires. Le député SPD Haase, celui-là même qui avait annoncé en août 14 le vote par son parti des crédits de guerre, réclame l'abolition de la monarchie. Le Reichstag le conspue. Mais il tient bon. 4. Le prince Max de Bade, 
qui a quitté le Reichstag aussitôt après son discours, rentre chez lui, malade de la grippe.

Épisode V: Dans les appartements du prince Max de Bade. Le prince, très souffrant, tente de continuer à diriger les affaires. Il essaye d'obtenir l'abdication de Guillaume II dans le but de sauver la monarchie en faisant monter sur le trône l'un de ses fils. Il apprend que le Kaiser, sans l'avoir consulté, part pour Spa, où se trouve le Grand QuartierGénéral, avec l'intention de demander aux chefs de l'armée de l'aider à se maintenir au pouvoir. Rétrospection : Max de Bade fait un cauchemar : il revoit en rêve les erreurs et les crimes de l'impérialisme allemand. Au réveil, on lui communique que les démarches entreprises pour convaincre Guillaume II d'abdiquer ont échoué.

Épisode VI : Du côté des humbles. 1. Trude Müller, la femme de Max Müller, dont la mort sous un tank était la scène initiale (cf. I, 1), coud des manteaux de soldat dans sa chambre, située dans une " caserne locative » de Berlin. Son fils, âgé de cinq ans, est à l'hôpital avec une pneumonie. 2. Trude Müller va faire la queue aux abattoirs, où est annoncée une distribution de viande. Elle y rencontre Lucie Lange, sa voisine, fiancée d'un matelot de la flotte de guerre basée à Kiel. Mécontentement de la population allemande affamée. En rentrant chez elle, Trude apprend que son fils est mort. 2. Trude Müller se rend à l'hôpital pour voir le cadavre de son enfant. 4. Le père de Lucie Lange, Wilhelm Lange, se rend à son travail à l'usine AEG. C'est un militant confirmé du SPD. Il est furieux contre le fiancé de sa fille, qui tient des propos favorables aux bolcheviks. Il déteste aussi les «braillards » de l'USPD. Il se remémore son passé de militant dévoué et se réjouit à l'idée que le SPD va enfin arriver au pouvoir. Description réaliste de son travail à l'usine, où il est chef d'équipe au marteau-pilon. Il lui faut écouter les récriminations de ses collègues, mécontents de la façon dont se comportent les «bonzes » de SPD. 5. A la centrale thermique de Rummelsburg. Entre les militants SPD et USPD règne une certaine tension. Monologue intérieur du technicien Suit, membre de l'USPD: il fait le procès du SPD. Il est tenté de rejoindre l'organisation des «Revolutionäre Obleute», comme le lui conseille vivement son collègue Primelsack. 6 . Une semaine plus tard: Primelsack et son camarade font l'essai d'une bombe. 7 . (29.10.1918) Réunion des Revolutionäre Obleute, à laquelle vient assister Liebknecht. Portrait de Karl Liebknecht. Il expose ses idées sur la stratégie révolutionnaire.

Épisode VII : La mutinerie des marins de Kiel. 1. (29.10.1918) La flotte reçoit l'ordre d'appareiller. Le gros de la flotte est encore à l'ancre à Wilhelmshaven. Le lecteur est transporté dans la chaufferie du cuirassé «Grosser Kurfürst». Il assiste au pénible travail des chauffeurs qui viennent de recevoir la consigne de régler la température des chaudières pour une allure de douze milles. Nous faisons connaissance avec le chauffeur Raumschuh, le fiancé de Lucie Lange. Soudain, la nouvelle se répand que la flotte doit appareiller pour une action offensive. Fureur des marins, qui croyaient la paix imminente. 2. Sur le pont du «Grosser Kurfürst», où est monté le chauffeur Jakob Bonczyk. Quelques camarades le rejoignent. Ils aperçoivent, peu distinctement car il fait encore nuit, la masse d'un autre cuirassé, qui semble dériver : c'est le «König Albert». Il envoie un message lumineux en morse: les chauffeurs ont éteint les chaudières. Sur la passerelle, le commandant du «Grosser Kurfürst» repasse mentalement l'opération à mener : il s'agit d'engager la bataille avec la flotte britannique, de manière à susciter l'enthousiasme de la population et à faire échouer, simultanément, les négociations d'armistice. On lui transmet le message en provenance du «König Albert»; il donne l'ordre de garder la nouvelle secrète. Mais elle est déjà 
diffusée dans tout le navire. Sabotage également à bord du «Markgraf». La mutinerie éclate à bord du "Grosser Kurfürst». Le chauffeur Raumschuh en prend la tête. 3. (30.10.1918) Le commandement de la flotte décide de réagir. Réunion des amiraux sur le cuirassé «Baden» : ils décident de réprimer la révolte. 4. (31.10.1918) On procède à des arrestations. Seul Raumschuh reconnaît avoir participé à la mutinerie. 5. Les navires dont les équipages se sont mutinés sont ramenés à Kiel. Internement des mutins. Puis on tente de les disperser en les acheminant vers d'autres villes de l'Allemagne du nord. 6. (02.11.1918) Un contingent de marins est acheminé sur Kiel par Hambourg. 7. (03.11.1918) Le dimanche à Kiel. Les permissionnaires se répandent dans la ville. Les patrouilles ont reçu des consignes pour être moins sévères que d'habitude. Atmosphère générale de contestation dans les estaminets. Une assemblée générale des marins au «Exerzierplatz» se prépare. Les officiers tentent d'empêcher qu'elle ait lieu en déclenchant une "alerte générale ». Mais l'atmosphère a déjà atteint un tel degré d'effervescence que les marins n'obéissent plus. 8. Le terrain d'exercice en bordure de ville. Des syndicalistes tentent de prendre le contrôle du mouvement, mais ils sont débordés. Les marins n'ont plus confiance dans les chefs locaux des partis de gauche. La foule des marins se porte vers les prisons et délivre les camarades incarcérés. Mais la garde ouvre le feu : 8 morts et 29 blessés. Les marins refluent en désordre. 9. Noske arrive à Kiel, dépêché par Ebert, et réussit à prendre sous son contrôle le mouvement des marins. Intervention de l'instance narrative: rappel des idées de Noske, personnalité fondamentalement hostile à la révolution. Son habileté tactique: il parvient à convaincre les marins que leur mouvement, pour être efficace durablement, doit être dirigé. En même temps, il prend des contacts avec le Secrétaire d'Etat impérial à la Marine. Noske est présenté comme le social-démocrate typique, celui qui sait faire avorter les mouvements de grève en prenant leur direction. Duplicité de Noske. Duplicité de la social-démocratie en général. Raisons pour lesquelles la stratégie de Noske a réussi.

Épisode VIII: Les marins propagent la révolution. 1. Le Sénat (gouvernement) de la ville libre de Hambourg accepte de négocier avec les Conseils de travailleurs et de soldats. 2. Le suicide du grand armateur Albert Ballin, figure légendaire du capitalisme hambourgeois et interlocuteur écouté de Guillaume II 3. Le départ des marins pour Berlin. Ils se font intercepter en gare de Celle. Mais les marins entraînent les soldats dans leur révolte et prennent le contrôle de la gare. 4. (08.11.1918) A Braunschweig, les masses en révolte, entraînées par cinq marins, contraignent le duc de Brunswick à l'abdication. Peinture des masses en mouvement. La révolution spontanée, 5. (9 novembre 1918 au matin). Les marins en route pour Berlin. Ils y sont arrêtés dès leur arrivée.

30 Épisode IX: La révolution de novembre à Berlin. 1. (8 novembre 1918, 10 heures du matin) Réunion du gouvernement, toujours dirigé par Max de Bade, en conseil de guerre. Bilan de la situation: la révolte est en train de gagner toute l'Allemagne. Pour neutraliser l'emprise croissante de l'USPD, le SPD est amené à durcir sa position. L'empereur doit partir, dit Scheidemann, si l'on veut éviter la révolution. 2. Réunion du groupe parlementaire SPD au Reichstag. Les sociaux-démocrates majoritaires votent une motion demandant au prince Max de Bade de demeurer chancelier, du moins jusqu'à la conclusion de l'armistice. 3. Réunion de l'USPD, mais vu la présence suspecte d'indicateurs de police devant l'immeuble du Schiffbauerdamm où se trouve le bureau des socialistes indépendants, ceux-ci décident de se réunir au Reichstag. 4. Montée de l'élan révolutionnaire dans les usines de Berlin. Le Conseil des travailleurs et des 
soldats de Berlin lance un appel à la grève générale et à la manifestation. Il exige que soit proclamée la République socialiste. 5. A la Chancellerie du Reich : le prince Max de Bade téléphone à la Villa Fraineuse à Spa, résidence temporaire de Guillaume II, pour le pousser à abdiquer au plus vite. Mais le Kaiser fait traîner les choses. Le prince Max tente désespérément de sauver le régime monarchique malgré l'aveuglement de l'empereur. 6. Les ouvriers sortent des usines pour manifester. Les militants du SPD essayent de freiner le mouvement. On voit reparaître le père de Lucie Lange : membre actif du SPD depuis 22 ans, Wilhelm Lange se voit accuser avec véhémence d'être un traitre à la classe ouvrière. Les soldats refusent de tirer sur la foule et passent aux insurgés. 7. Retour à la Chancellerie. L'annonce de l'abdication se fait attendre. Le gouvernement apprend que les soldats de la garnison de Berlin sont en train de passer du côté des insurgés. 8. Le Kaiser au "front ", en fait à la Villa Fraineuse à Spa. Groener lui fait un bilan de la situation militaire, qui est désastreuse, le Kaiser tente d'obtenir le soutien de l'armée pour continuer de régner. Groener l'informe qu'il ne doit plus y compter. Groener a été le gouverneur militaire de la place de Kiev : il a pu se rendre compte en Russie de la force d'impact de l'idéologie communiste. Sa préoccupation essentielle est la lutte contre le péril bolchevik. Il connaît bien Ebert, et sait que ce dernier partage son point de vue. Les sociaux-démocrates majoritaires seront les alliés de Groener dans sa lutte contre le bolchévisme. Arrivée du Kronprinz. Attitude dilatoire de Guillaume II : il consent à abdiquer en tant qu'Empereur d'Allemagne, mais non en tant que Roi de Prusse. 9. Berlin, Chancellerie du Reich, 9.11.1918. Le gouvernement du prince Max de Bade continue d'attendre avec une impatience grandissante qu'on lui notifie depuis Spa une déclaration d'abdication sans ambiguïté. Une délégation socialiste conduite par Ebert demande à être reçue. Ebert demande le pouvoir; il admet néanmoins que quelques représentants des partis bourgeois pourront être membres (en qualité de Secrétaires d'État et à titre de conseillers techniques) du nouveau gouvernement qu'il envisage de former. Les troupes stationnées à Berlin étant passées du côté des Conseils, Max de Bade ne voit plus d'autre solution que de céder le pouvoir à la gauche modérée et propose à Ebert le poste de Chancelier. Ebert s'assure préalablement de la coopération de l'USPD. Textes des proclamations adressées par Ebert aux autorités et aux citoyens.

Épisode X : «Hier Groener». La conclusion du pacte fatidique entre Ebert et Groener. (Journée du 9 novembre 1918). 1. Les masses berlinoises se mettent en mouvement, prenant possession de la rue. Discours de Liebknecht proclamant l'Allemagne République socialiste. Les spartakistes occupent le Reichstag. Atmosphère de confusion bon enfant. Proclamation (préventive) de la République par Scheidemann. Fureur de Ebert. Les masses occupent le Palais Impérial (Schloss) ; pillage des réserves alimentaires, qui sont d'un luxe et d'une profusion inconvenants. Discours de Liebknecht depuis le balcon d'où parlait Guillaume II ; il lance un appel à la Révolution mondiale. Enthousiasme des masses. 2. Les adieux du prince Max de Bade à Ebert. 3. La population de Berlin en marche pour défendre la révolution. 4 . Ebert cherche à obtenir le ralliement de l'USPD et des spartakistes, un accord pour une ligne d'action commune. Liebknecht refuse de coopérer, mais l'USPD ne se démarque pas aussi nettement. 4. Réapparition du malheur individuel : Trude Müller cherche vainement son mari dans une gare parmi la foule des soldats qui reviennent $d u$ front. 5. Ebert, resté seul dans son bureau, passe mentalement en revue les raisons qui vont le décider à faire appel au soutien des militaires, des hauts cadres, autrefois abhorrés, de l'armée impériale. La sonnerie du 
téléphone retentit : Groener est à l'appareil. Ebert accepte son offre de collaboration, et les conditions dont elle est assortie.

Ce découpage confirme les vues de Susan Suleiman, selon qui la «redondance » est un "critère formel générique du roman à thèse " ${ }^{9}$. Les séquences événementielles présentent un caractère " anaphorique » : elles répètent une leçon présentée avec des focalisations variées, mais dont le contenu idéel demeure identique d'un bout à l'autre du texte. La redondance interprétative (le message: le SPD a trahi le prolétariat) instaure l'unité d'un processus complexe fait autant de malentendus, de hasards malencontreux, d'occasions manquées que de volonté d'action.

Ce qui empêche la redondance de devenir intolérable ${ }^{10}$, c'est que les valeurs sont en fin de compte problématisées. Le point de vue exposé par Plievier est hostile aux compromis funestes conclus par le SPD, et sans complaisance à l'égard d'un vouloir socialiste présenté comme inauthentique. Mais Plievier n'est pas non plus tout à fait favorable aux éléments spartakistes. A la fin du livre, le sens manifeste, que l'on croyait surabondamment démontré, se dérobe. On voit même Liebknecht sur le point de se rallier au projet de cabinet paritaire de Friedrich Ebert, tout en posant des conditions extravagantes (p. 371). L'œuvre relève donc de la pratique littéraire, malgré tout; et cela dans la mesure où elle ne campe pas de personnages totalement positifs. En outre, il demeure une béance, due à l'inachèvement du processus décrit. Il y a là un trait constitutif fondamental du roman d'actualité ${ }^{11}$. La sensation de l'inachèvement contraste avec l'intensification du réel, dont la brusque interruption lors de la clôture a pour but de faire surgir une angoisse que peut seule guérir l'action politique.

\section{NOTES}

1. - Le récit mimétique est basé sur une convention, un pacte de vraisemblance avec le lecteur : l'auteur fait comme si le monde fictionnel pouvait appartenir à la réalité empirique. Le récit de Plievier utilise le pacte avec un signe inversé : on a l'impression que la période historique décrite appartient à un monde fictionnel.

2. - Plievier, Der Kaiser blieb, die Generäle blieben, éd. Fischer Taschenbuch Verlag, 1981. «Nachwort», p. 373-374.

3. - La distinction qui paraît s'imposer entre le roman historique et le roman d'actualité n'a pas toujours une valeur opératoire. Le roman historique est toujours, au fond, un texte qui reflète les préoccupations du moment où il est écrit. Les problèmes contemporains sont la substance véritable du roman historique. Cf. L. Feuchtwanger, Das Haus der Desdemona oder Grösse und Grenzen der historischen Dichtung, Fischer Taschenbuch Verlag, 1986, p. 135).

4. - Cf. Harry Wilde, Theodor Plievier. Nullpunkt der Freiheit, Verlag Kurt Desch, München, Wien, Basel, 1965, p. 260-261.

5. - La simplification des décors témoigne d'une volonté de dépouillement qui s'accorde avec la simplicité des émotions collectives. Cf. p. 299 : «Uber dem Hafen liegt ein dunkler Regenhimmel. Die Umrisse eines grossen Krans und des Reihenstiegsschwimmdocks am anderen Ufer zeichnen sich im Dunst ab. Alles andere ist weggewischt».

Les notations chronologiques sont tout aussi sommaires : «Einen Tag später, am 17. Okt. 1918» 
(p. 29) ; «Eine Woche vergeht» (p. 105) ; «29. Oktober 1918! Am selben Abend, an dem der Kaiser von Berlin ins Hauptquartier flüchtete, zur selben Stunde, da Karl Kiebknecht in der Sitzung der revolutionären Obleute das Wort nahm und zum revolutionären Massenkampf aufforderte, erhielt die deutsche Hochseeflotte den Befehl zum Auslaufen». (p. 121). La nature du récit impose une temporalité strictement chronologique et surtout la multiplicité des repères pour souligner l'ordre événementiel. En revanche, il n'y a pratiquement aucune indication de durée intime.

6. - Cf. Susan R. Suleiman, Le roman à thèse ou l'autorité fictive, P.U.F., Paris, 1983.

7. - Suleiman, op. cit., p. 179.

8. - Cité par L. Goldmann, Pour une sociologie du roman, coll. «Idées », Gallimard, Paris, 1964, p. 153.

9. - Suleiman, op. cit., cf. le chapitre intitulé «Classification des types de redondance possibles dans le roman réaliste», p. 198-207. La citation est p. 186.

10. - Suleiman, op. cit., p. 235 sq., sur le problème de la « redondance excessive ».

11. - Cf. A. Lagny, Personnages juifs dans le Zeitroman, dans Germanica, Univ. de Lille III, $\mathrm{n}^{\circ} 9$, «Die Neue Sachlichkeit - Lebensgefühl oder Markenzeichen?», p. 146: «Le Zeitroman, genre de l'époque ouverte, $d u$ sens qui n'est pas encore constitué, ne se réduit pas à une variante $d u$ roman historique. Celui-ci en effet prend pour cadre une époque révolue, close sur elle-même, dont la lisibilité lui permet de figurer esthétiquement la résolution d'une problématique, même contemporaine ».

\section{RÉSUMÉS}

Cette contribution examine le roman Der Kaiser ging, die Generäle blieben : dans quelle mesure est-il un exemple valable pour ce qui touche à la transformation esthétique de l'actualité ? Du point de vue de la forme, l'œuvre est une relation de la révolution manquée de novembre 18 à la manière d'un reportage rédigé au présent: elle montre un événement qui appartient déjà à l'histoire, mais qui reste actuel parce qu'il continue d'agir sur la réalité contemporaine. Le but recherché est de créer un sentiment d'urgence civique. C'est pourquoi la reconstitution de l'événement fait appel aux émotions, et n'est pas simplement un exercice intellectuel comme c'est le cas pour l'histoire scientifique ou pseudo-objective. Cela nécessite l'utilisation de procédés que l'on peut qualifier dans l'ensemble de reportage fictionnalisé et de séquence filmique. La vérité poétique ainsi produite est plutôt néo-objectiviste que marxiste à proprement parler.

Dieser Beitrag prüft die Eignung von Der Kaiser ging, die Generäle blieben als Demonstrationsobjekt für die ästhetische Umsetzung des Zeitgeschehens. Der Plievier-Roman hat die Form einer im Präsens geschriebenen Berichterstattung über die verfehlte Novemberrevolution: er bringt bereits Geschichtliches, aber auch fortwirkende Zeitwirklichkeit. Als Effekt soll ein Bewusstsein der staatsbürgerlichen Dringlichkeit entstehen, daher werden die Vorgänge auch emotional, und nicht nur intellektuell im Sinne einer objektiven oder pseudoobjektiven Historiographie nachgebildet. Dies erfordert den Gebrauch von formalen Mitteln, die sich insgesamt unter den Bezeichnungen fiktive Reportage und filmische Sequenzen subsumieren lassen. Die dadurch erzeugte poetische Wahrheit ist eher eine neusachliche als eine regelrecht marxistische zu nennen. 
AUTEUR

PIERRE VAYDAT

Université Charles-de-Gaulle - Lille III 